\title{
Emotion, entropy evaluations and subjective uncertainty
}

\author{
Lara Bertram (lara.bertram@surrey.ac.uk) \\ University of Surrey, Psychology Deptartment, Guildford, UK \\ Eric Schulz (eric.schulz@ tuebingen.mpg.de) \\ Max Planck Institute for Biological Cybernetics, Tübingen, Germany \\ Matthias Hofer (mhofer@mit.edu) \\ Massachusetts Institute of Technology, Cambridge, Massachusetts, USA \\ Jonathan D. Nelson (j.d.nelson@surrey.ac.uk) \\ University of Surrey, Psychology Deptartment, Guildford, UK
}

\begin{abstract}
A variety of conceptualizations of psychological uncertainty exist. From an information-theoretic perspective, probabilistic uncertainty can be formalized as mathematical entropy. Cognitive emotion theories posit that uncertainty appraisals and motivation to reduce uncertainty are modulated by emotional state. Yet little is known about how people evaluate probabilistic uncertainty, and about how emotional state modulates people's evaluations of probabilistic uncertainty and behavior to reduce probabilistic uncertainty. We tested intuitive entropy evaluations and entropy reduction strategies across four emotion conditions in the Entropy Mastermind game. We used the unified Sharma-Mittal space of entropy measures to quantify participants' entropy evaluations. Results suggest that many people use a heuristic strategy, focusing on the number of possible outcomes, irrespective of the probabilities in the probability distribution. This result is surprising, given that previous work suggested that people are very sensitive to the maximum probability when choosing queries on probabilistic classification tasks. Emotion induction generally increased participants' heuristic assessment. The uncertainty associated with emotional states also affected game play: participants needed fewer queries and spent less time on games in high-uncertainty than in low-uncertainty emotional states. Yet entropy perceptions were not related to subjectively reported uncertainty, numeracy or entropy knowledge, suggesting that entropy perceptions may form an independent psychological construct.
\end{abstract}

Keywords: Entropy; human entropy intuitions; Sharma-Mittal space; emotion; uncertainty

\section{Introduction}

Uncertainty is a fundamental characteristic of the world and a key concept in theories of cognition and emotion. In cognitive theories of the value of information, mathematical entropy quantifies the uncertainty about a variable given a probability distribution. Uncertainty can be reduced by acquiring information, quantified as information gain (Lindley et al., 1956; Oaksford \& Chater, 1994; Crupi, Nelson, Meder, Cevolani, \& Tentori, 2018). Many different entropy models for quantifying probabilistic uncertainty have been proposed. Even though Shannon entropy is widely used (Coenen, Nelson, \& Gureckis, 2018; Fuhrman et al., 2000; Martignon, Von Hassein, Grün, Aertsen, \& Palm, 1995; Oaksford \& Chater, 1994), other entropy measures also are important in some contexts. Quadratic entropy (Lande, 1996) is important in ecology; the families of Rényi entropies (Rényi et al., 1961) have proven powerful in computer science and image processing; Tsallis entropies (Tsallis, 2011) are widely used in physics. Bayes's error is another kind of entropy (Crupi et al., 2018), widely used in various domains. Despite the centrality of probabilistic uncertainty in models of the value of information, which model best captures human intuitions about probabilistic uncertainty is an open question. Moreover, most previous work has considered how people assess the information value of possible questions, rather than the perception of probabilistic uncertainty itself.

Furthermore, research on cognition and emotion on people's uncertainty perceptions have to this point remained separate from each other, despite their shared theoretical basis. Cognitive theories of emotion define uncertainty as a cognitive component characterizing emotional states (Smith \& Ellsworth, 1985; Scherer, Schorr, \& Johnstone, 2001). For example, anger and pride are characterized by low uncertainty appraisals, whereas anxiety and curiosity are characterized by high uncertainty appraisals. Previous research has investigated the role of emotion in judgment, risk assessment, and decision making under uncertainty. It was found that emotion-related uncertainty appraisals modulate risk assessment and information processing (Tiedens \& Linton, 2001; Lerner \& Keltner, 2001). In these studies, psychological uncertainty is usually assessed using questionnaires which ask respondents about their subjective appraisal of uncertainty in a situation (Smith \& Ellsworth, 1985). This conceptualization of psychological uncertainty lacks precise quantification and mathematical formalisation. Entropy offers such a mathematically precise quantification of uncertainty, yet how emotions, appraisals of uncertainty and entropy perceptions are related is an open empirical question. Furthermore, little research (Hirsh, Mar, \& Peterson, 2012, is an exception) has looked at the role of different characteristics of emotion in basic assessments of probabilistic uncertainty and in behavior directed towards uncertainty reduction.

Our research has three goals: quantifying human intuitions about probabilistic uncertainty in the Sharma-Mittal space of entropy measures; investigating the relationship between entropy and emotion-theoretical conceptualizations of psychological uncertainty; and investigating which characteristics of emotions best predict emotion effects on assessments of prob- 
abilistic uncertainty and behavior aimed at reducing probabilistic uncertainty.

\section{Emotions, uncertainty and information}

Cognitive emotion theories state that emotions are characterized by cognitive appraisals (Smith \& Ellsworth, 1985; Scherer et al., 2001). The Appraisal Tendency Framework (Lerner \& Keltner, 2000) posits that high-uncertainty emotional states are associated with increased perceptions of risks, leading to more systematic information processing and decreased risk-seeking behavior (Tiedens \& Linton, 2001; Lerner \& Keltner, 2001; Wright \& Bower, 1992; Smith \& Ellsworth, 1985). Other theories of emotion make diverging predictions: Feelings-as-information theory (Schwartz, Woloshin, Black, \& Welch, 1997) conceptualizes emotions as a source of information about the situation and predicts that negative affective states will motivate effortful, systematic and analytical thinking, whereas positive affective states will lead to more heuristic, effortless information processing. The valence of an emotion is also hypothesized to affect probability and risk estimates (Wright \& Bower, 1992), with negative moods making estimates more negative and positive moods influencing assessments in a positive direction. The emotioncognition relationship can also be interpreted in terms of cognitive load. In this view, emotions pull resources away from cognitive tasks and thus interfere with cognitive processing (Plass \& Kalyuga, 2019). Emotions may also have the function to guide the allocation of attention (Derryberry \& Tucker, 1994; Wichary, Mata, \& Rieskamp, 2016).

\section{The Sharma-Mittal space of entropy measures}

The Sharma-Mittal space of entropy measures offers a framework in which different entropy models arise in a unified parametric space (Crupi et al., 2018; Sharma \& Mittal, 1977). Each entropy model describes the entropy in a random variable $K$, which can take values $k_{1}$ through $k_{n}$. We can envision $K$ to be an object's unknown category, and $k_{1}$ through $k_{n}$ to be possible categories. Two key ideas are important for characterizing an entropy function in the Sharma-Mittal space (Fig. 1 ), each of which is associated with a particular parameter.

One idea is how surprising it would be if a particular event $k_{i}$ were to happen, i.e., if the object were to be category $k_{i}$. Surprise is maximal if the prior probability of the event $P\left(k_{i}\right)=0$, and null if $P\left(k_{i}\right)=1$. Different entropy functions have different maxima, and differently shaped surprise curves, but surprise is always a nonincreasing (and typically a monotonically decreasing) function of $P\left(k_{i}\right)$. Sharma-Mittal entropy uses the Tsallis (1988) family of surprise functions, determined by a degree parameter $t$ in the vertical axis. Existing data on people's query selection, and ratings of possible queries' usefulness, place strong constraints on psychologically plausible degree parameter values (Crupi et al., 2018).

The other key idea is that entropy is average surprise, but that rather than averaging the raw surprise values themselves, individual surprise values are passed through an averaging function $g$, as a self-weighted entropy (Crupi et al., 2018) in the General Theory of Means (Muliere \& Parmigiani, 1993) framework. The order parameter $r$ determines the averaging function in the Sharma-Mittal space.

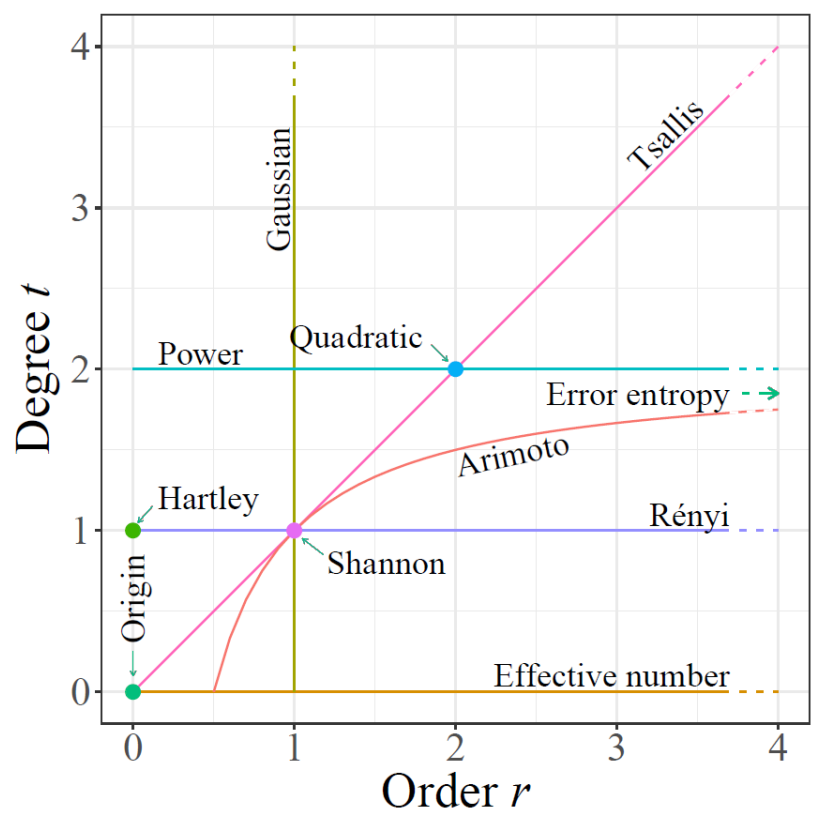

Figure 1: Sharma-Mittal space of entropy measures. (Crupi et al., 2018)

The order parameter $r$ determines the ranking of different probability distributions' uncertainty, which may vary in number of possibilities as well as their individual probabilities (Crupi et al., 2018). For most values of $r$, the entropy of a distribution depends on all of the probabilities in that distribution. However, if $r$ is infinite, the entropy depends only on the largest probability in the distribution. In the other extreme case of $r=0$, entropy is a function of the number of "live" (greater-than-zero-probability) possibilities. This can be viewed as a heuristic model because of its computational simplicity; it can also be called "balanced" in the sense that it treats all possibilities equally, irrespective of their probability. For instance, entropy models with order about 1.51 deem a $50 \%, 50 \%$ distribution to be equal in entropy to a $85 \%, 5 \%$, $5 \%, 5 \%, 5 \%, 5 \%$ distribution. Higher-order entropy models deem the $50 \%, 50 \%$ distribution to be higher entropy; lower-order entropy models deem the six-item distribution to be higher entropy. The most psychologically plausible order parameter value is not well constrained by existing empirical data; hence, we focus on it here.

\section{Entropy Mastermind}

Studying people's subjective assessments of uncertainty requires making the idea of probabilistic uncertainty understandable. We employed Entropy Mastermind for this purpose. Entropy Mastermind (Figure 2) is a single-player appbased game in which the computer generates a secret code by drawing from a known, and typically nonuniform, probability distribution, represented iconically as a "fruit bowl". The 


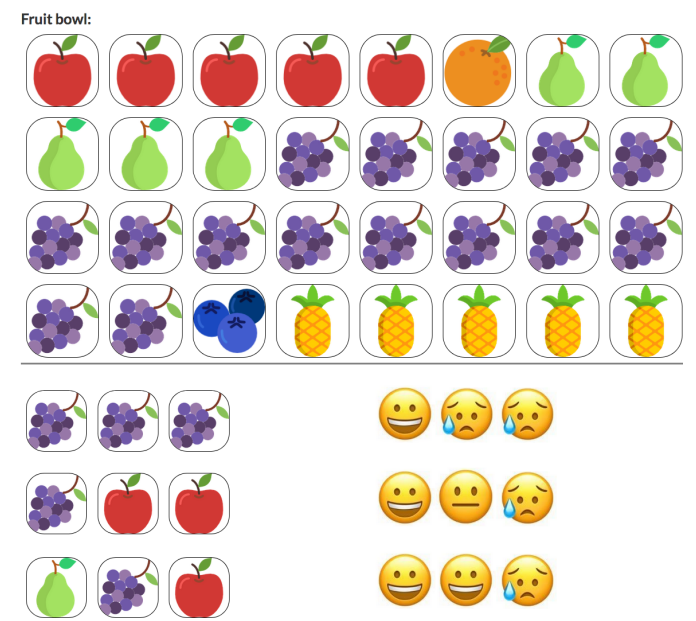

Figure 2: Entropy Mastermind. Top: Icon array presenting an example fruit bowl that generated the hidden fruit code. Probability distributions follow one of four entropy recipes, resulting in low, medium low, medium high and high (shown) entropy levels. Codes are generated by random sampling with replacement. The player guesses which fruit is in each position of the three slots of the secret code, by clicking on the position they want to change. The participant then clicks a "Check" button (not shown), to receive feedback. Bottom: Game play. The first guess is 3 grape items. The feedback (one smiling and two frowning faces) conveys that exactly one of the items is correct in kind and location. However, the player does not know which of the three items is in the correct position: In the feedback, smiley faces always come first, then neutral faces, and lastly frowning faces. In the second guess, the player tests grape in the first position, and apple in the other positions. The feedback (smiling, neutral, frowning face) indicates that one item is correct in kind and location, another item is in the code but needs to be moved, and another item is not in the code at all. From the third guess, the player can infer that grape is in the middle, apple on the right, and pear is not in the code. Game play continues until the hidden code is fully identified.

items are mixed before each draw, and items of the code are drawn with replacement. The player has to guess the secret code by making queries (testing possible codes), and getting feedback in the form of smileys (happy, neutral, sad). The number of queries needed is a measure of the difficulty to break the code. In empirical data from a previous experiment, the number of queries needed to break the code was an increasing function of the entropy in the generating distribution (Schulz, Bertram, Hofer, \& Nelson, 2019).

To quantify the order parameter describing people's intuitive assessment of relative uncertainty in probability distributions, we asked participants questions about the relative difficulty of playing Mastermind with two differently entropic code jars. Difficulty was defined as number of queries participants expected to need to break the secret code. Response patterns across four such questions (Figure 3) allow mapping of participants onto the order parameter of the SharmaMittal space. Note that this mapping arises from the pattern of responses in the four comparisons, not single responses or aggregate scores.

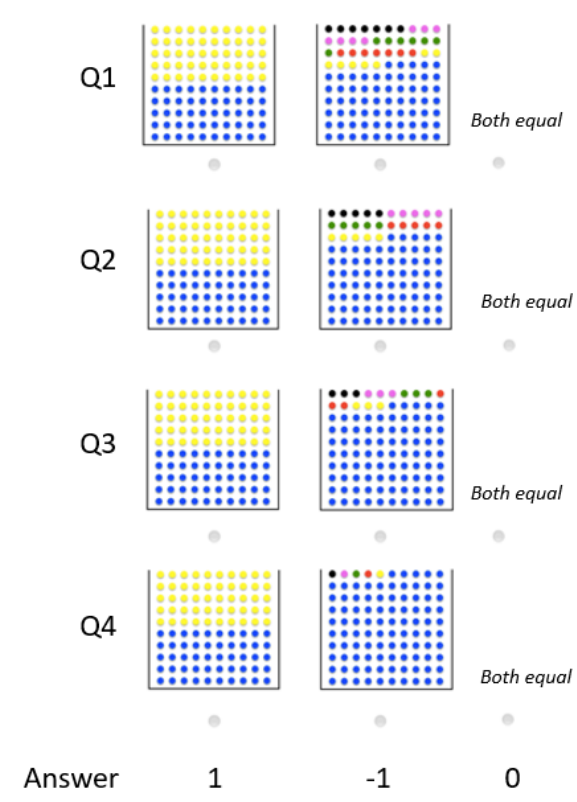

Figure 3: Entropy Intuitions. Question: Which code jar would be harder/easier to play Mastermind with? Answer Options: The $50 \%, 50 \%$ jar/the 6 item jar/both are equal. Answers place respondents along the order parameter axis of the Sharma-Mittal space.

\section{Towards a theory of human entropy perceptions}

Previous research on information search behavior in a probabilistic visual classification task, in which probabilities were learned from experience, suggested that probability gain, which is the expected reduction in Bayes's error (Nelson, McKenzie, Cottrell, \& Sejnowski, 2010), is a psychologically plausible entropy model. Bayes's error has an infinite order parameter; related high-order entropy models are also consistent with experimental data to date (Crupi et al., 2018). However, people's valuation of information on words-andnumbers tasks gives a wide range of possible order parameter values (Crupi et al., 2018). Because of the contradictory findings in previous work, and the fact that asking people the usefulness of queries (as in previous work) is several steps removed from assessing uncertainty itself, we did not have strong hypotheses about the results of the present study. Rather, we designed the stimuli with the goal to establish whether people's entropy intuitions, in the context of this task, are consistent with any Sharma-Mittal entropy models, and to be able to discriminate among a wide range of order parameter values.

\section{Method}

Materials and Procedure 109 first-year undergraduate psychology students (99 female, $M_{\mathrm{age}}=19.39 ; S D=2.65$; range: 18 to 39 ) at University of Surrey participated in our study as part of a cognitive psychology class. Informed consent was given in accordance with the University's procedures and the Helsinki Declaration. Students were first introduced to the rules and interface of the game. Participants 


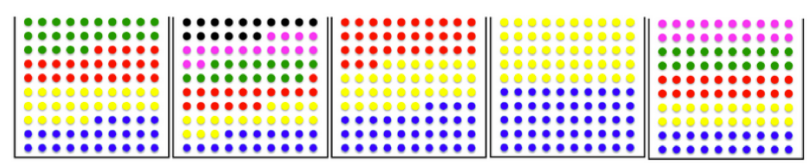

Figure 4: Entropy Axioms Question Select the code jar which would make the game as easy/hard as possible (the easier/harder the game, the less/more queries are needed). Correct answer: $50 \%, 50 \%=$ easiest; 6 -color jar $=$ hardest. Entropy increases with the number of different items if items are uniformly distributed.

were randomly assigned to one of 5 conditions: writing about an episode of anger, pride, anxiety, curiosity or a neutral event (control). Emotions differed in valence (positive: curiosity, pride; negative: anxiety, anger) and uncertainty (high uncertainty: anxiety, curiosity; low uncertainty: anger, pride). Participants completed a set of questionnaires, including a test of numeracy, uncertainty appraisals and questions testing intuitions about entropy. After a brief emotion refresh, participants played 12 rounds of Entropy Mastermind.

Questionnaires and entropy questions Numeracy was assessed using the adaptive Berlin Numeracy Test (Cokely, Galesic, Schulz, Ghazal, \& Garcia-Retamero, 2012), combined with 3 additional items from (Schwartz et al., 1997) to cover a wider range of numeracy levels. The results of both tests were added, forming an aggregate numeracy score ranging from 1 to 7 . Uncertainty appraisals were assessed using a 12-item short form of the Intolerance of Uncertainty questionnaire (Carleton, Norton, \& Asmundson, 2007) and the Dimensional Ratings Scale (Smith \& Ellsworth, 1985). The latter was slightly adapted to assess uncertainty appraisals in the current situation instead of appraisals in the situation reported during the emotion manipulation. Entropy knowledge questions tested participants' understanding of key axioms of entropy. These are axioms that, up to a possible tie, would be satisfied not only by all Sharma-Mittal entropy measures, but by virtually any entropy measures in the literature (Csiszár, 2008). For example, one such axiom holds that if each possibility is equally probable, a probability distribution with a greater number of possible outcomes is higher entropy. To test understanding of this axiom (Figure 4), participants were asked which of five uniform probability distribution code jars, ranging from 2 to 6 colors of marbles, would be hardest for playing Entropy Mastermind. We administered these questions to assess potential effects of numeracy and entropy knowledge on entropy assessments and control for these effects. We designed four questions $(Q 1-Q 4$ in Figure 3 ) to test participants' intuitions about the relative entropy in different code jars. These questions asked participants which of two code jars would be harder to play with. Response patterns on these questions, if consistent with the formalism, place participants along the order parameter axis (typically in a range) of the Sharma-Mittal space (Figure 1). A 1 coded the answer that the $50 \%, 50 \%$ distribution is harder to play with, 0 that both distributions are equally hard to play with and -1 that the distribution containing 6 different item types is harder to play with. The response patterns, derived from the combined responses in $Q 1, Q 2, Q 3$ and $Q 4$, and associated order parameters are found in Table 1 .

Entropy Mastermind gameplay Participants were required to correctly answer four questions about of the rules of the game before starting. Participants were instructed to attempt to guess the secret code with as few guesses as possible. Participants played 12 rounds of Mastermind. In each game, one of four entropy conditions was randomly selected, resulting in a corresponding fruit bowl icon array. The secret fruit code was generated from this fruit distribution. In the very high entropy condition, the proportions were $(5,5,5,5,6,6)$, in the high entropy condition $(1,1,5,5,5,15)$, in the low entropy condition $(1,1,1,4,4,21)$ and in the very low entropy condition, the secret code was sampled from the proportions $(1,1,1,1,1,27)$.

\section{Results}

In a first step, the order parameter characterizing each participant was inferred from answer patterns on the four entropy intuitions questions (Figure 3, following conversion rules in Table 1). Out of the $3^{4}=81$ possible response patterns, only nine are consistent with the Sharma-Mittal formalism. $75.2 \%$ of participants responded to the questions in a consistent way. We followed a strict exclusion criterion and labeled any response pattern inconsistent with the 9 consistent patterns as undefined, irrespective of their potential proximity to a meaningful response. For example, the answer pattern -1001 shows a clear ranking of code jars, yet the two "equal" rankings would not allow for this participant to be mapped onto the order parameter axis of the Sharma-Mittal space.

\section{Distribution of participants across order parameters}

A $\chi^{2}$ test revealed a significant deviation from a uniform distribution of participants across order parameters, both when including undefined $\left(\chi^{2}=158.8, d f=9, p<.001, V=0.4\right)$, and excluding undefined answers $\left(\chi^{2}=158.37, d f=8, p\right.$ $<.001, V=0.49)$. Overall, the largest group of participants (38.53\%) answered the entropy intuitions questions following an order parameter of $0-0.55$. This response pattern always deems the six item distribution to be higher in entropy than the 50\%,50\% distribution. This behavior can be interpreted as a heuristic strategy in which only the number of different kinds of items in the distribution are counted and the relative proportions of each type are discarded. A learner whose intuitions correspond to Origin entropy, for which $r=t=0$, would respond in this way. The second-largest group among the consistent response patterns (13.76\%), was formed by participants with an order parameter of $.56-0.96$, rating the 6-item distribution as higher entropy, except for in $Q 4$. This response pattern still focuses mainly on the number of possibilities, yet pays some attention to the individual probabili- 
Table 1: Ranking of response patterns and corresponding order parameters.

\begin{tabular}{|c|c|c|c|c|c|c|c|c|c|}
\hline Response Pattern & $-1-1-1-1$ & $-1-1-10$ & $-1-1-11$ & $-1-101$ & $-1-111$ & -1011 & -1111 & 0111 & 1111 \\
\hline Rank & 1 & 2 & 3 & 4 & 5 & 6 & 7 & 8 & 9 \\
\hline Nr Participants & 42 & 4 & 15 & 0 & 12 & 1 & 2 & 0 & 6 \\
\hline Order Parameter & $0-.55$ & $.55-.56$ & $.56-.96$ & $.96-.97$ & $.97-1.51$ & $1.51-1.52$ & $1.52-2.58$ & $2.58-2.59$ & $2.59-\infty$ \\
\hline
\end{tabular}

ties. This group was succeeded by $11 \%$ of participants with order parameter $.97-1.51$, who estimated the 6 item distribution to be higher in entropy in $Q 1$ and $Q 2$ and the 50\%,50\% distribution as higher in entropy in $Q 3$ and $Q 4$. Order ranks $2,4,6$, and 8 deemed exactly one of the 6-item distributions equal to the $50 \%, 50 \%$ distribution. These ties were rarely used. The number of participants per order parameter and the corresponding order parameter intervals (upper and lower bound) are presented in Table 1.

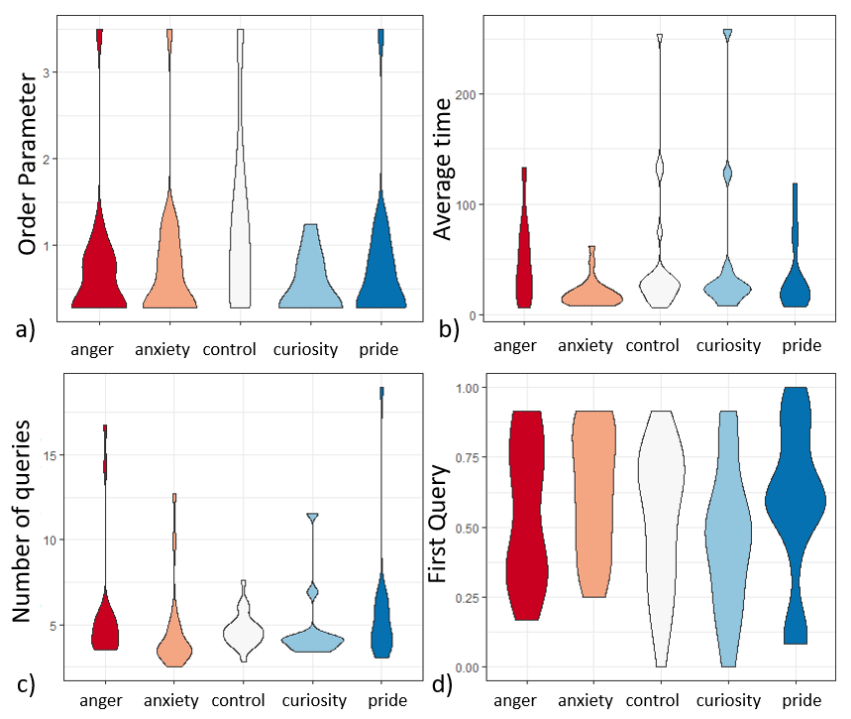

Figure 5: Distribution of order parameters and game play variables by emotion condition. a) Order parameter by emotion condition. b) Average time to solve a game in seconds by emotion condition. c) Average number of queries per game by emotion condition. d) Probability to select most probable items only in the first query by emotion condition.

We conducted bootstrapped ANOVAs to analyse participants' intuitive entropy assessments. Respondents with undefined answer patterns were excluded from analyses, resulting in a sample size of $N=82$.

\section{Entropy evaluations, numeracy and entropy knowledge}

To test whether entropy intuitions were predicted by numeracy (cumulative numeracy score from the Berlin Numeracy test and the Schwarz et al. questions) and entropy knowledge (cumulative answer scores on questions such as in Figure 4), a bootstrapped ANOVA with order parameter as dependent variable and numeracy and entropy knowledge as independent variables was conducted. Neither numeracy $(p=.94)$ nor entropy knowledge $(p=.2)$ predicted entropy intuitions, suggesting that differences between entropy types cannot be explained by differences in numeracy or entropy knowledge. To test whether numeracy and entropy knowledge predicted whether respondents' answer patterns were consistent with the Sharma-Mittal formalism, we conducted nonparametric Wilcoxon tests between the defined and undefined groups of participants. We found that respondents in the defined group had higher entropy knowledge $(M=3, S D=1.05)$ than respondents in the undefined group $(M=2.56, S D=0.87 ; \mathrm{W}$ $=1378, p=.05, d=.47)$. Groups did not differ in numeracy $(p=.59)$.

\section{Entropy Mastermind game play}

As the entropy intuitions questions were based on the Mastermind game, we were interested in the relationship between participants' intuitive entropy perceptions and subsequent game play behavior. Particularly, we wanted to test whether number of queries needed, time spent on games and the composition of first guesses were related to the entropy order parameter of respondents. We conducted bootstrapped ANOVAs with order parameter as predictor and a) Reaction Time (average time spent on games in seconds), b) Queries (average number of queries needed to break the code per game) and c) $1_{s t}$ Query All Most Probable (probability that respondent's first guess is exclusively comprised of items of the type which dominates the distribution) as dependent variables. Results were not significant (all $p>.05$ ), indicating that entropy intuitions and basic game play parameters are not related to the order parameter characterizing people's entropy assessments.

\section{Emotions, entropy evaluations and entropy reduction}

Next, we looked at the effects of the emotion manipulation on subjective entropy assessments. In a bootstrapped ANOVA, the effect of emotion condition on order parameter was significant $(p=.04)$. Closer examination of distributions revealed that the main difference in order parameter was driven by the difference between the control condition and the emotion conditions (see Figure 5, top left). This was confirmed by a bootstrapped ANOVA with the binary variable emotion vs. control as predictor and order parameter as dependent variable $(p=.003)$. The emotion manipulation pushed participants' order parameter estimates towards more heuristic low-order assessments of entropy. Interestingly, differences between groups in game play showed a more nuanced picture: Independent Wilcoxon tests revealed that the two uncer- 
tain emotion groups (anxiety and curiosity) differed significantly from the two certain emotion groups (pride and anger) in average number of queries needed per game $(W=344, p$ $=.01, d=0.27)$ and average time spent on games in seconds ( $W=386, p=.04, d=0.34$ ), but not time spent on individual queries $(p=.26)$. On average, respondents in the high uncertainty conditions needed fewer queries $(M=4.64, S D$ $=2.4)$ and spent less time on games $(M=31.5, S D=47.12)$ than those in the low uncertainty conditions $(M=5.71, S D$ $=3.65$ and $M=42.81, S D=36.36$ ). These differences were not significant when grouping conditions based on valence, i.e. when comparing the two positive (curiosity, pride) and the two negative (anxiety, anger) emotions (all $p>.05$ ). The probability to select only the most probable items in the first query was not affected by emotion.

To investigate the relationship between uncertainty appraisals and probabilistic uncertainty assessments, we tested whether people's order parameter was predicted by subjectively reported uncertainty appraisals. We also tested whether basic assumptions of cognitive emotion theories, i.e. effects of emotions on uncertainty appraisals, were reproduced in our experiment. Contradicting appraisal-based emotion theories, bootstrapped ANOVAs revealed no effect of emotion condition on uncertainty appraisals $(p=.59)$ and no difference in uncertainty appraisals between the high and low uncertainty emotion conditions $(p=.26)$. Neither were subjective uncertainty appraisals correlated with assessments of probabilistic uncertainty $(p=.75)$.

\section{Discussion}

In this study, we investigated how people intuitively evaluate the relative entropy of probability distributions in different emotional states. Our research provides novel evidence on people's basic perceptions of probabilistic uncertainty.

We found that the majority of participants $(75.2 \%)$ showed a response pattern consistent with the Sharma-Mittal formalism and could be positioned along the order axis of the space. This gives reason to assume that for most people the SharmaMittal space is an adequate formalism to capture entropy perceptions. Yet participants with little knowledge about entropy, as measured with the entropy axiom questions, were less likely to give consistent answers. This indicates that a basic understanding of entropy axioms promotes intuitions that are consistent with any Sharma-Mittal entropy metric. The most common response pattern suggested an order parameter between 0 and 0.55 . This response pattern can be interpreted as a heuristic, in which entropy is largely or solely evaluated based on the count of items in the distribution, with larger counts indicating higher entropy, irrespective of the relative proportions. The second largest $(13.8 \%$, order parameter between 0.56 and 0.96) and third largest (order parameter between 0.97 and 1.51) groups of respondents also followed a relatively low order entropy assessment, with the number of items still prominent in the evaluation. Emotion induction increased respondents' tendency to use this heuristic strat- egy, supporting cognitive load theory. In all four emotional states, irrespective of uncertainty and valence characterizing the emotion, participants' order parameters were lower than in the control group.

The low order parameter we found is surprising, as it is not only less than found in previous research (Crupi et al., 2018; Nelson et al., 2010), but in the first two largest groups even below Shannon entropy. Among the possible explanations of our findings are attentional processes which favor count over proportion and which may be promoted by emotional arousal. Future work could address the nature of the attentional processes at work during entropy assessments in emotional and non-emotional states by using eye tracking. Key questions could be whether subjects in emotional and non-emotional states differ in gaze direction, attention allocation to different parts of the six-item probability distributions and between the two-item and six-item probability distributions. A prediction in line with our findings would be that the increased cognitive load in emotional states leads to more heuristic information processing as fewer resources are available, manifesting in increased attention allocation to low-probability items and less eye movement (and thus fewer comparisons) between code jars. Motivation could also play a role: it requires relatively low-effort to rate all the 6-item distributions as higher in entropy than the $50 \%, 50 \%$ distribution. As students participated in our study as part of the lecture without credit or payment for participation, they may have had little incentive for performing well on the task. Future work could test whether incentivising participants changes entropy evaluations.

Despite the general low-order entropy intuitions we found, participants' responses varied widely, including even 2.59 to $\infty$ order responses. These responses might reflect meaningful individual differences. Among the potential modulators of entropy assessments are individual differences related to uncertainty perceptions, such as neuroticism, trait anxiety, and psychological disorders associated with altered uncertainty evaluations. Another interesting research question is whether entropy intuitions are modulated by experience. In our study, respondents' answers were based on visual information about the probability distributions only. Assessing entropy intuitions after having played Mastermind with differently entropic code jars could reveal changes in assessments due to experience. In this context, testing diverse populations differing in experience with probabilities and entropy, for example students in mathematical versus non-mathematical subjects, and at different ages, would shed light on the role of experience, expertise and cognitive development in the formation of entropy intuitions over the life course.

Our findings suggest that the general tendency to employ a low order heuristic strategy was increased by the induction of emotions. One interpretation of this finding is that experiencing emotions detracts capacities from cognitive tasks. Interestingly though, this general effect did not show in game play, where the difference between high and low uncertainty emotions predicted people's time spent on queries and average 
number of queries needed, not emotion per se. Surprisingly, we did not find an effect of emotion condition on subjective uncertainty appraisals. This contradicts previous work within the Appraisal Tendency Framework and other cognitive emotion theories. One explanation could be that we rephrased items measuring uncertainty appraisals to capture uncertainty appraisals in the current situation instead of appraisals associated with the emotional episode reported in the emotion manipulation. As cognitive emotion theories predict that the cognitive effects of emotions carry over to situations unrelated to the emotional event, we expected to find an effect of emotions on uncertainty appraisals reported directly after the emotion manipulation. Our findings challenge this core assumption of cognitive emotion theories. Yet we did find an effect of uncertainty associated with the induced emotion on game play: respondents in high uncertainty emotional states needed fewer queries and less time to solve games than respondents in low uncertainty emotional states. One could interpret this finding in terms of increased motivation to reduce uncertainty in high uncertainty emotional states. Our mixed findings highlight the need for further research on the carryover effect of emotions and associated cognitive processes on unrelated situations, and on the relationship between entropy, uncertainty appraisals and emotions. This research could then feed into the development of a comprehensive theory of the role of incidental emotions in different kinds of uncertainty assessments.

Summing up, we found that a surprisingly low order parameter best described participants' evaluations of the relative entropy of different probability distributions. This suggests that in our task, many participants focused exclusively or almost-exclusively on the number of possibilities, largely or completely disregarding the overall probability distribution. This tendency was increased by emotion induction. Uncertainty associated with people's emotional state modulated game play. More research is required to validate and extend our findings, exploring psychological variables associated with entropy intuitions and the role of experience, expertise and age in the formation of entropy intuitions. Adding questions quantifying respondents' degree parameter and modelling of game play data could extend our model of human entropy intuitions and give insight into the relationship between behavioural entropy reduction strategies and entropy intuitions.

\section{References}

Carleton, R. N., Norton, M. P. J., \& Asmundson, G. J. (2007). Fearing the unknown: A short version of the intolerance of uncertainty scale. Journal of Anxiety Disorders, 21, 105-117.

Coenen, A., Nelson, J. D., \& Gureckis, T. M. (2018). Asking the right questions about the psychology of human inquiry: Nine open challenges. Psychonomic Bulletin \& Review, 26, 15481587.

Cokely, E. T., Galesic, M., Schulz, E., Ghazal, S., \& GarciaRetamero, R. (2012). Measuring risk literacy: The berlin numeracy test. Judgment and Decision Making, 7, 25-47.

Crupi, V., Nelson, J. D., Meder, B., Cevolani, G., \& Tentori, K. (2018). Generalized information theory meets human cognition:
Introducing a unified framework to model uncertainty and information search. Cognitive Science, 42, 1410-1456.

Csiszár, I. (2008). Axiomatic characterizations of information measures. Entropy, 10, 261-273.

Derryberry, D., \& Tucker, D. M. (1994). Motivating the focus of attention. In P. M. Niedenthal \& S. Kitayama (Eds.), The heart's eye: Emotional influences in perception and attention (pp. 167196). Academic Press.

Fuhrman, S., Cunningham, M. J., Wen, X., Zweiger, G., Seilhamer, J. J., \& Somogyi, R. (2000). The application of shannon entropy in the identification of putative drug targets. Biosystems, 55, 5 14.

Hirsh, J. B., Mar, R. A., \& Peterson, J. B. (2012). Psychological entropy: A framework for understanding uncertainty-related anxiety. Psychological Review, 119, 304-320.

Lande, R. (1996). Statistics and partitioning of species diversity, and similarity among multiple communities. Oikos, 5-13.

Lerner, J. S., \& Keltner, D. (2000). Beyond valence: Toward a model of emotion-specific influences on judgement and choice. Cognition \& emotion, 14, 473-493.

Lerner, J. S., \& Keltner, D. (2001). Fear, anger, and risk. Journal of personality and social psychology, 81, 146-159.

Lindley, D. V., et al. (1956). On a measure of the information provided by an experiment. The Annals of Mathematical Statistics, 27, 986-1005.

Martignon, L., Von Hassein, H., Grün, S., Aertsen, A., \& Palm, G. (1995). Detecting higher-order interactions among the spiking events in a group of neurons. Biological cybernetics, 73, 69-81.

Muliere, P., \& Parmigiani, G. (1993). Utility and means in the 1930s. Statistical Science, 421-432.

Nelson, J. D., McKenzie, C. R., Cottrell, G. W., \& Sejnowski, T. J. (2010). Experience matters: Information acquisition optimizes probability gain. Psychological Science, 21, 960-969.

Oaksford, M., \& Chater, N. (1994). A rational analysis of the selection task as optimal data selection. Psychological Review, 101, 608-631.

Plass, J. L., \& Kalyuga, S. (2019). Four ways of considering emotion in cognitive load theory. Educational Psychology Review, 31, 339-359. Retrieved from https://doi.org/10.1007/s10648 $-019-09473-5$

Rényi, A., et al. (1961). On measures of entropy and information. In Proceedings of the fourth berkeley symposium on mathematical statistics and probability, volume 1: Contributions to the theory of statistics.

Scherer, K. R., Schorr, A., \& Johnstone, T. (2001). Appraisal processes in emotion: Theory, methods, research. Oxford University Press.

Schulz, E., Bertram, L., Hofer, M., \& Nelson, J. (2019). Exploring the space of human exploration using entropy mastermind. Proceedings of the 2019 Conference of the Cognitive Science Society, 2762-2768

Schwartz, L. M., Woloshin, S., Black, W. C., \& Welch, H. G. (1997). The role of numeracy in understanding the benefit of screening mammography. Annals of Internal Medicine, 127, 966-972.

Sharma, B. D., \& Mittal, D. P. (1977). New non-additive measures of relative information. Journal of Combinatorics Information \& System Sciences, 2, 122-132.

Smith, C. A., \& Ellsworth, P. C. (1985). Patterns of cognitive appraisal in emotion. Journal of Personality and Social Psychology, 48, 813-838.

Tiedens, L. Z., \& Linton, S. (2001). Judgment under emotional certainty and uncertainty: the effects of specific emotions on information processing. Journal of personality and social psychology, 81,973 .

Tsallis, C. (1988). Possible generalization of boltzmann-gibbs statistics. Journal of Statistical Physics, 52, 479-487.

Tsallis, C. (2011). The nonadditive entropy sq and its applications in physics and elsewhere: Some remarks. Entropy, 13, 1765-1804.

Wichary, S., Mata, R., \& Rieskamp, J. (2016). Probabilistic inferences under emotional stress: How arousal affects decision processes. Journal of Behavioral Decision Making, 29, 525-538.

Wright, W. F., \& Bower, G. H. (1992). Mood effects on subjective probability assessment. Organizational Behavior and Human Decision Processes, 52, 276-291. 